\title{
Nonlocal vibration of embedded double-layer graphene nanoribbons in in-phase and anti-phase modes
}

\author{
Jin-Xing Shi ${ }^{a}$, Qing-Qing Ni ${ }^{\text {b,c* }}$, Xiao-Wen Lei ${ }^{a}$, Toshiaki Natsuki ${ }^{b}$ \\ ${ }^{a}$ Interdisciplinary Graduate School of Science \& Technology, Shinshu University, 3-15-1 Tokida, Ueda, \\ Nagano 386-8567, Japan \\ ${ }^{b}$ Department of Functional Machinery \& Mechanics, Shinshu University, 3-15-1 Tokida, Ueda, Nagano \\ 386-8567, Japan \\ ${ }^{c}$ Key Laboratory of Advanced Textile Materials and Manufacturing Technology Ministry of Education, \\ Zhejiang Sci-Tech University, Hangzhou 310018, P. R. China
}

\section{Abstract}

Graphene nanoribbons (GNRs), the finite-wide counterparts of crystalline graphene sheets, have been potential materials used in nano-devices because of their excellent electronic, thermal and mechanical properties. The vibrational property of GNRs embedded in an elastic matrix is important to study in the application of GNRs. In this work, a theoretical analysis of nonlocal elasticity theory for the free vibrational characteristics of embedded double-layer GNRs (DLGNRs) is proposed based on continuum and Winkler spring models. We find two types of vibrational modes, in-phase mode (IPM) and anti-phase mode (APM), by a mathematical method. The results show that the natural frequencies of DLGNR embedded in an elastic matrix are significantly influenced by vibrational modes, nonlocal effects, the aspect ratio of DLGNRs and the Winkler foundation modulus.

*Corresponding author:

E-mail: niqq@shinshu-u.ac.jp; Tel: +81-268-21-5438; Fax: +81-268-21-5438 


\section{Keywords:}

Graphene nanoribbons; Nonlocal elasticity theory; Vibration; In-phase mode; Anti-phase mode.

\section{Introduction}

A conceptual model named graphite ribbon was first developed by Fujita et al. in 1996 [1], when they investigated the electronic states of graphite ribbons with armchair and zigzag edges by performing tight binding band calculations. Graphene sheet (GS), an infinite two-dimensional layer consisting of $\mathrm{sp}^{2}$ hybridized carbon atoms, was produced in 2004 [2]. Since then, graphite ribbon, often called graphene nanoribbon (GNR) that possesses a large aspect ratio [3], has attracted significant interests because of its remarkable electronic $[4,5]$, thermal $[6,7]$ and mechanical properties [8-16].

A wide range of methods to produce GNRs is available. Kosynkin et al. [17] and Cataldo et al. [18] successfully synthesized GNRs by oxidative unzipping of carbon nanotubes (CNTs). Cai et al. [19] devised a simple, bottom-up approach to produce GNRs with different topologies and widths. Sen et al. [20] produced GNRs by tearing GSs from adhesive substrates and discovered the formation of tapered GNRs. Fan et al. [21] fabricated GNRs by a microexplosion method, which involved filling multi-walled CNTs 
with potassium and then reacted them vigorously with water.

Vibration occurs during certain manufacturing processes (e.g., ultrasonication in nanocomposite processing) and as part of some nondestructive evaluation processes (e.g., Raman spectroscopy) [22, 23]. Investigation of the vibrational characteristics of materials is thus of fundamental importance for understanding electron transport in electron devices and is also of general interest [8]. Vibrational properties play a role in structural stability, structure identification and ballistic transport through electron-phonon coupling [9]. Gillen et al. [10] investigated the symmetry property of the vibrational modes of GNRs by using density functional theory (DFT), and their calculations suggested that the frequency splitting of the fundamental longitudinal-optical and transverse-optical modes exhibits a characteristic family dependence with quasi-metallic GNRs. Rao et al. [24] pointed out that double-layer GSs are of considerable interest as well as single-layer GSs. Moreover, double-layer GNRs (DLGNRs) has been proposed as the only semiconductor to produce insulating state and switch-off electrical conduction [25] and Behfar et al. [26] pointed out that a graphite sheet or a multi-layered graphene sheet is used in polymer composites as embedded structures to enhance its strength. Therefore, it is important to study the vibrational characteristics of DLGNRs embedded in an elastic matrix for the practical applications. However, this kind of research has been seldom reported to our 
knowledge.

On the other hand, nonlocal effects often become prominent at nanometer scales, the cause of which needs to be explicitly addressed with the general increasing interest in nanotechnology. In this study, the natural frequencies of DLGNRs embedded in an elastic matrix are analyzed by nonlocal elasticity theory based on a continuum model. In the theoretical approach, we divide the vibrational mode into in-phase mode (IPM) and anti-phase mode (APM), which is a feature of DLGNRs [27, 28]. The influence of vibrational modes (IPM and APM), nonlocal parameters, the aspect ratio of the DLGNRs and the Winkler foundation modulus on the natural frequencies of embedded DLGNRs are investigated in detail and compared with our previous work [29].

\section{Theoretical approach}

GNRs can be treated as a continuum model $[12,13]$ which is mostly used in theoretical analysis. Fig. 1(a) shows a continuum model of the DLGNRs, in which $L$ and $b$ are the length and width of the DLGNRs, respectively. The upper and lower layers of the DLGNRs are coupled to each other by van der Waals (vdW) interaction forces.

\subsection{Governing equations}

Nonlocal elastic theory has been used in mechanical analysis of nanoscale materials, 
such as CNTs [30-32] and GSs [33-38]. In especial, Pradhan et al. [35, 36] and Ansari et al. $[37,38]$ studied the vibration characteristics of orthotropic GSs and multilayer GSs based on nonlocal plate model. Whereas for the vibration analysis of GNRs, a special kind of GSs with a large aspect ratio, the nonlocal beam model is preferred. Because of the Euler-Bernoulli beam theory is a simple but practical tool for validating the beam deflection calculation, we can divide IPM and APM directly and provide an appropriate method for the further application of DLGNRs. Based on the nonlocal beam model, the Hooke' s law of GNRs can be determined as a nonlinear relationship given as

$$
\sigma-\left(e_{0} a\right)^{2} \frac{\partial^{2} \sigma}{\partial x^{2}}=E \varepsilon
$$

where $\sigma$ and $\varepsilon$ are the axial stress and axial strain, respectively. $E$ is the elastic modulus of GNRs. $e_{0} a$ is the nonlocal parameter appropriate to GNRs, where $e_{0}$ is a constant that is appropriate to the material, and $a$ is the characteristic internal length of a $\mathrm{C}-\mathrm{C}$ bond, which is $0.142 \mathrm{~nm}$. It should be noted that the value of $e_{0}$ needs to be determined from experiments or by matching dispersion curves of plane wave with those of atomic lattice dynamics, which has not yet been achieved for GNRs and remains an unresolved problem currently.

The definitions of the resultant bending moment $M$ and the kinematic relationship in a continuum beam model are considered, that is 


$$
\begin{gathered}
M(x, t)=\int_{A} z \sigma d A \\
\varepsilon=-z \frac{\partial^{2} w(x, t)}{\partial x^{2}}
\end{gathered}
$$

where $x, z$ are the coordinates shown in Fig. 1 (a). $t$ is time. $w$ is the flexural deflection of

GNRs and $A$ is the area of the cross-section of the GNRs. Then Eq. (1) can be rewritten as

$$
M(x, t)-\left(e_{0} a\right)^{2} \frac{\partial^{2} M(x, t)}{\partial x^{2}}=-E I \frac{\partial^{2} w(x, t)}{\partial x^{2}}
$$

where $I$ is the moment of inertia of the GNRs.

Based on the Euler-Bernoulli beam model, which assumes that the cross-section of a

DLGNR remains planar during flexion and is perpendicular to the neutral axis, we obtain

$$
\begin{gathered}
\frac{\partial V(x, t)}{\partial x}=\rho A \frac{\partial^{2} w(x, t)}{\partial t^{2}}-p(x) \\
V(x, t)=\frac{\partial M(x, t)}{\partial x}
\end{gathered}
$$

where $V$ is the resultant shear force. $\rho$ is the mass density of the GNRs. $p$ is the distributed transverse pressure acting on a DLGNR per unit of axial length.

Using Eqs. (4)-(6), the governing equation of motion for a continuum beam is derived as

$$
E I \frac{\partial^{4} w(x, t)}{\partial x^{4}}+\rho A \frac{\partial^{2} w(x, t)}{\partial t^{2}}-\left(e_{0} a\right)^{2}\left[\rho A \frac{\partial^{4} w(x, t)}{\partial x^{2} \partial t^{2}}-\frac{\partial^{2} p(x)}{\partial x^{2}}\right]=p(x)
$$

For the upper and lower layers of a DLGNR, Eq. (5) can be rewritten as the following two-coupled equations,

$$
E I \frac{\partial^{4} w_{1}}{\partial x^{4}}+\rho A \frac{\partial^{2} w_{1}}{\partial t^{2}}-\left(e_{0} a\right)^{2}\left[\rho A \frac{\partial^{4} w_{1}}{\partial x^{2} \partial t^{2}}-\frac{\partial^{2} p_{1}}{\partial x^{2}}\right]=p_{1}
$$




$$
E I \frac{\partial^{4} w_{2}}{\partial x^{4}}+\rho A \frac{\partial^{2} w_{2}}{\partial t^{2}}-\left(e_{0} a\right)^{2}\left[\rho A \frac{\partial^{4} w_{2}}{\partial x^{2} \partial t^{2}}-\frac{\partial^{2} p_{2}}{\partial x^{2}}\right]=p_{2}
$$

where the subscripts 1 and 2 denote the quantities associated with the upper and lower layers of a DLGNR, respectively.

The Winkler spring model has been widely used to analyze the mechanical properties of embedded GSs [38], in which the elastic matrix is described as a Winkler model characterized by the spring. The cross-section of a DLGNR embedded in an elastic matrix is shown in Fig. 1 (b), in which the Winkler foundation modulus relative to the elastic matrix is defined as $k_{W}$. Then the distributed transverse pressure acting on the upper and lower layers of a DLGNR can be given by

$$
\begin{aligned}
& p_{1}=c\left(w_{1}-w_{2}\right)+k_{W} w_{1} \\
& p_{2}=c\left(w_{2}-w_{1}\right)+k_{W} w_{2}
\end{aligned}
$$

where $c$ is the vdW interaction coefficient between the upper and lower layers, which can be obtained from the Lennard-Jones pair potential [39], and is given as

$$
\begin{gathered}
c=-b\left(\frac{4 \sqrt{3}}{9 a}\right)^{2} \frac{24 \zeta}{\delta^{2}}\left(\frac{\delta}{a}\right)^{8}\left[\frac{3003 \pi}{256} \sum_{k=0}^{5} \frac{(-1)^{k}}{2 k+1}\left(\begin{array}{l}
5 \\
k
\end{array}\right)\left(\frac{\delta}{a}\right)^{6} \frac{1}{\left(\overline{z_{1}}-\overline{z_{2}}\right)^{12}}\right. \\
\left.-\frac{35 \pi}{8} \sum_{k=0}^{2} \frac{(-1)^{k}}{2 k+1} \frac{1}{\left(\overline{z_{1}}-\overline{z_{2}}\right)^{6}}\right]
\end{gathered}
$$

where $\zeta=2.968 \mathrm{meV}$ and $\delta=3.407 \AA$ are parameters chosen to fit the physical properties of GNRs, and $\bar{z}_{j}=z_{j} / a(j=1,2)$, where $z_{j}$ is the coordinate of the $j$ th layer in the 
direction of thickness with the origin at the midplane of the GNR.

To divide the vibration modes into IPM and APM, we assume

$$
\begin{aligned}
& y_{1}=w_{1}+w_{2} \\
& y_{2}=w_{1}-w_{2}
\end{aligned}
$$

From Eqs. (8) and (9), the governing equations of IPM and APM can be derived as, respectively,

$$
\begin{gathered}
E I \frac{\partial^{4} y_{1}}{\partial x^{4}}+\rho A \frac{\partial^{2} y_{1}}{\partial t^{2}}-\left(e_{0} a\right)^{2}\left(\rho A \frac{\partial^{4} y_{1}}{\partial x^{2} \partial t^{2}}-k_{W} \frac{\partial^{2} y_{1}}{\partial x^{2}}\right)=k_{W} y_{1} \\
E I \frac{\partial^{4} y_{2}}{\partial x^{4}}+\rho A \frac{\partial^{2} y_{2}}{\partial t^{2}}-\left(e_{0} a\right)^{2}\left[\rho A \frac{\partial^{4} y_{2}}{\partial x^{2} \partial t^{2}}-\left(2 c+k_{W}\right) \frac{\partial^{2} y_{2}}{\partial x^{2}}\right]=\left(2 c+k_{W}\right) y_{2}
\end{gathered}
$$

Considering that the deflection of a DLGNR has different vibration modes $Y_{j}(x), j=1$,

2 for the IPM and APM, respectively, the displacements of the vibration solution in the DLGNRs can be given by

$$
y_{j}=Y_{j}(x) e^{i \omega t} \quad j=1,2
$$

where $\omega$ is the vibrational frequency of DLGNRs.

Substituting Eq. (17) into Eqs. (15) and (16), the governing equations of the vibrational properties for the IPM and APM of DLGNRs embedded in an elastic matrix are obtained as

$$
\begin{aligned}
& E I \frac{\partial^{4} Y_{1}}{\partial x^{4}}+\left(e_{0} a\right)^{2} H_{1} \frac{\partial^{2} Y_{1}}{\partial x^{2}}-H_{1} Y_{1}=0 \\
& E I \frac{\partial^{4} Y_{2}}{\partial x^{4}}+\left(e_{0} a\right)^{2} H_{2} \frac{\partial^{2} Y_{2}}{\partial x^{2}}-H_{2} Y_{2}=0
\end{aligned}
$$


where

$$
\begin{gathered}
H_{1}=\rho A \omega^{2}+k_{W} \\
H_{2}=\rho A \omega^{2}+2 c+k_{W}
\end{gathered}
$$

2.2 Solution of governing equations

By solving the four-order polynomial functions Eqs. (18) and (19), the whole solution of the governing equations can be obtained as follows

$$
Y_{1}(x)=C_{1} \cos \left(\lambda_{1} x\right)+C_{2} \sin \left(\lambda_{1} x\right)+C_{3} e^{\lambda_{2} x}+C_{4} e^{-\lambda_{2} x}
$$

and

$$
Y_{2}(x)=C_{5} \cos \left(\lambda_{3} x\right)+C_{6} \sin \left(\lambda_{3} x\right)+C_{7} e^{\lambda_{4} x}+C_{8} e^{-\lambda_{4} x}
$$

or

$$
Y_{2}(x)=C_{5} e^{\lambda_{5} x}+C_{6} e^{-\lambda_{5} x}+C_{7} e^{\lambda_{4} x}+C_{8} e^{-\lambda_{4} x}
$$

$$
\text { or } Y_{2}(x)=e^{\lambda_{6} x}\left[C_{5} \cos \left(\lambda_{7} x\right)+C_{6} \sin \left(\lambda_{7} x\right)\right]+e^{-\lambda_{6} x}\left[C_{7} \cos \left(\lambda_{7} x\right)+C_{8} \sin \left(\lambda_{7} x\right)\right]
$$

where $C_{m}(m=1,2, \ldots, 8)$ are the coefficients that need to be determined via boundary

conditions, and

$$
\begin{aligned}
& \lambda_{1}=\sqrt{\frac{\left(e_{0} a\right)^{2} H_{1}+\sqrt{\left(e_{0} a\right)^{4} H_{1}^{2}+4 E I H_{1}}}{2 E I}} \\
& \lambda_{2}=\sqrt{\frac{-\left(e_{0} a\right)^{2} H_{1}+\sqrt{\left(e_{0} a\right)^{4} H_{1}^{2}+4 E I H_{1}}}{2 E I}} \\
& \lambda_{3}=\sqrt{\frac{\left(e_{0} a\right)^{2} H_{2}+\sqrt{\left(e_{0} a\right)^{4} H_{2}^{2}+4 E I H_{2}}}{2 E I}} \\
& \lambda_{4}=\sqrt{\frac{-\left(e_{0} a\right)^{2} H_{2}+\sqrt{\left(e_{0} a\right)^{4} H_{2}^{2}+4 E I H_{2}}}{2 E I}}
\end{aligned}
$$




$$
\begin{aligned}
& \lambda_{5}=\sqrt{\frac{-\left(e_{0} a\right)^{2} H_{2}-\sqrt{\left(e_{0} a\right)^{4} H_{2}^{2}+4 E I H_{2}}}{2 E I}} \\
& \lambda_{6}=\sqrt{\frac{\left(e_{0} a\right)^{2} H_{2}+2 \sqrt{-E I H_{2}}}{4 E I}} \\
& \lambda_{7}=\sqrt{\frac{-\left(e_{0} a\right)^{2} H_{2}+2 \sqrt{-E I H_{2}}}{4 E I}}
\end{aligned}
$$

\subsection{Boundary conditions}

The boundary conditions for IPM and APM of free embedded DLGNRs of length $L$ are given as

$$
\begin{aligned}
& \frac{d^{2} Y_{1}(0)}{d x^{2}}=\frac{d^{2} Y_{1}(L)}{d x^{2}}=\frac{d^{3} Y_{1}(0)}{d x^{3}}=\frac{d^{3} Y_{1}(L)}{d x^{3}}=0 \\
& \frac{d^{2} Y_{2}(0)}{d x^{2}}=\frac{d^{2} Y_{2}(L)}{d x^{2}}=\frac{d^{3} Y_{2}(0)}{d x^{3}}=\frac{d^{3} Y_{2}(L)}{d x^{3}}=0
\end{aligned}
$$

Substituting the deflection functions of the DLGNRs $\left(Y_{j}, j=1,2\right)$ into the boundary conditions, we obtain the simultaneous equations

$$
\begin{gathered}
\Omega[\omega, L]_{4 \times 4}\left[\begin{array}{l}
C_{1} \\
C_{2} \\
C_{3} \\
C_{4}
\end{array}\right]=0 \\
\Psi[\omega, L]_{4 \times 4}\left[\begin{array}{l}
C_{5} \\
C_{6} \\
C_{7} \\
C_{8}
\end{array}\right]=0
\end{gathered}
$$

where $\Omega[\omega, L]_{4 \times 4}$ and $\Psi[\omega, L]_{4 \times 4}$ are $4 \times 4$ matrices containing the natural frequency $\omega$ and the length $L$ of DLGNRs. The vibrational frequencies and associated vibration modes of DLGNRs embedded in an elastic matrix can be obtained from the 
eigenvalue $|\omega, L|_{4 \times 4}=0$, which is the condition of nontrivial solution of $C_{m}(m=1$, $2, \ldots, 8)$ in Eqs. (35) and (36).

\section{Analytical Results and Discussion}

To study the vibrational characteristics of DLGNRs embedded in an elastic matrix, each layer of a DLGNR is modeled as an individual classical thin beam of the same width, thickness and length. The effective thickness of each layer of a DLGNR is equal to the diameter of a carbon atom, $0.34 \mathrm{~nm}$. The aspect ratio of a DLGNR $L / b$ is larger than 5 because the Euler beam theory produces errors for structures with a small aspect ratio. The Young's modulus $E$ and mass density $\rho$ of the DLGNRs are the same as those of a GS; $1.02 \mathrm{TPa}$ and $2250 \mathrm{~kg} / \mathrm{m}^{3}$, respectively [39].

Based on the proposed theoretical approach, IPM and APM, derived from Eqs. (35) and (36), respectively, can occur in DLGNRs with free boundary conditions. As shown in Fig. 2, IPM 1-4 are the first four in-phase vibration modes of a DLGNR, which means that both the upper and lower layers have the same direction of deflection. APM 1-4 are the first four anti-phase vibration modes, in which the deflections of the upper and lower layers occur in the opposite direction. Unlike our previous work [29], the vibration amplitude ratios of the upper and lower layers in different vibrational modes are the same, 
because of the symmetry of the upper and lower layers of a DLGNR.

Fig. 3 shows the relationship between the natural frequencies $\left(k_{W}=0 \mathrm{GPa}\right)$ and vibrational modes of DLGNRs of different aspect ratio and $e_{0} a=0 \mathrm{~nm}$, where Figs. 3 (a) and (b) present the natural frequencies of IPM 1-4 and APM 1-4, respectively. It is clearly seen that as the number of the mode increases, the natural frequencies increase, and the shorter DLGNR show higher sensitivity to the mode number, which is the same as DWCNTs [29]. For example, the natural frequencies of DLGNRs for IPM 1-4 with $L / b=$ 10 ranges from 10.3 to $117.8 \mathrm{GHz}$, but for $L / b=20$ it is only 2.6 to $29.5 \mathrm{GHz}$. Furthermore, because of the influence of vdW interaction forces, when vibrations have the same harmonic number, the resonant frequencies of APM are larger than those of IPM.

The nonlocal effects on the natural frequencies of DLGNRs with $L / b=10$ for IPM $1-4$ and APM 1-4 as a function of the vibrational mode are shown in Figs. 4 (a) and (b), respectively. The natural frequencies of DLGNRs decrease with increasing nonlocal parameters $\left(e_{0} a\right)$, and, as both the numbers of the IPM and APM increase, the natural frequencies of DLGNRs become more sensitive to the nonlocal parameters. It is obvious that the natural frequencies of DLGNRs with different nonlocal parameters are nearly the same in both of the first mode of IPM and APM in Figs. 4 (a) and (b), and become 
scattered as the number of the vibrational mode increases.

To further study the nonlocal effects on the vibrational characteristics of DLGNRs, the nonlocal effects on the natural frequency of DLGNRs of different aspect ratio for the first mode of IPM are calculated, as shown in Fig. 5. The influence of nonlocal effects on the natural frequency lessen as the aspect ratio of DLGNR increasing, which is consistent with the findings in reference [40]. For example, when the aspect ratio $L / b$ of DLGNRs is 20, and $e_{0} a$ is assumed as $0,0.5,1$ and $2 \mathrm{~nm}$, the natural frequencies of DLGNRs are 2.57 2.56, 2.53 and $2.43 \mathrm{GHz}$, respectively, which are very similar to each other. In contrast, when aspect ratio $L / b=5$, and $e_{0} a=0,0.5,1$ and $2 \mathrm{~nm}$, the corresponding natural frequencies of DLGNRs are 41.05, 38.93, 34.12 and $24.66 \mathrm{GHz}$, respectively.

The relationship between frequencies and IPM and APM of DLGNRs embedded in different elastic matrices with $e_{0} a=0 \mathrm{~nm}$ and $L / b=10$ is shown in Fig. 6 . The influence of the surrounding elastic matrix on the natural frequency of DLGNRs is investigated based on the Winkler spring model. We take the ratio of the spring modulus to the vdW coefficient $\left(k_{W} / c\right)$ as a parameter to consider the stiffness of the elastic matrix. It can be found that for both IPM 1-4 and APM 1-4, when parameter $k_{W} / c$ is higher, the natural frequencies are higher, which means elastic matrix has a positive effect on the natural frequencies of both IPM and APM. The reason is that in both IPM and APM, DLGNRs 
are tightened by the elastic matrix, and it is well known that higher tightening causes higher frequency.

The natural frequencies of APM are less sensitive to the number of the vibration mode than those of the IPM as shown in Figs. 3, 4 and 6. This phenomenon is also found in DWCNTs [29], and the explanation is that the vdW interaction force is considered to be the dominating factor in frequency of APM but not in frequency of IPM.

\section{Conclusions}

In this study, the nonlocal effects on the free vibration of DLGNRs embedded in an elastic matrix were investigated. Based on the Euler-Bernoulli beam model, a theoretical approach was proposed to analysis the free vibration properties of DLGNRs with free supported boundary conditions. It is clarified that there exists two types of vibrational modes, IPM and APM, in which the deflections of the upper and lower layers occur in the same or opposite directions, respectively. The natural frequencies of DLGNRs are significantly influenced by the vibration mode (IPM and APM) and increase as the number of the mode increases. Nonlocal effects have a negative influence on the natural frequencies, especially for higher mode of IPM and APM. However, as the aspect ratio of the DLGNR increases, the nonlocal effects decrease until they become could be neglected. 
The Winkler foundation modulus has a positive influence on the natural frequency of both IPM and APM of DLGNRs embedded in an elastic matrix because higher tightening causes a higher frequency. Moreover, because of the influence of the vdW interaction forces, the natural frequencies of APM are less sensitive to the vibration modes than those of IPM. With above results, the proposed theoretical approach will provide a guidance in the application of embedded DLGNRs in dynamic mechanical analysis, high frequency oscillators, graphene-based electrochemical sensors and so on.

\section{Acknowledgments}

This work was supported by a Grant-in-Aid from the Global COE Program from the Ministry of Education, Culture, Sports, Science and Technology, and by CLUSTER (second stage) from the Ministry of Education, Culture, Sports, Science and Technology (Japan).

\section{References}

[1] M. Fujita, K. Wakabyashi, K. Nakada, K. Kusakabe, J. Phys. Soc. Jpn. 65 (1996) 1920.

[2] K.S. Novoselov, A.K. Geim, S.V. Morozov, D. Jiang, Y. Zhang, S.V. Dubonos, I.V. 
Grigorieva, A.A. Firsov, Science 306 (2004) 666.

[3] M. Terrones, A.R. Botello-Méndez, J. Campos-Delgado, F. López-Urías, Y.I. Vega-Cantú, F.J. Rodríguez-Macías, A.L. Elías, E. Muñoz-Sandoval, A.G. Cano-Márquez, J.C. Charlier, H. Terrones, Nano Today 5 (2010) 351.

[4] L. Yang, C.H Park, Y.W. Son, M.L. Cohen, SG. Louie, Phys. Rev. Lett. 99 (2007) 186801.

[5] O. Roslyak, G. Gumbs, D.H Huang, Phys. Lett. A 374 (2010) 4061.

[6] J. Campos-Delgado, Y.A. Kim, T. Hayashi, A. Morelos-Gómez, M. Hofmann, H. Muramatsu, M. Endo, H. Terrones, R.D. Shull, M.S. Dresselhaus, M. Terrones, Chem. Phys. Lett. 469 (2009) 177.

[7] J.W. Jiang, J.H Lan, J.S. Wang, B.W Li, J. Appl. Phys. 107 (2010) 054314.

[8] R. Gillen, M. Mohr, J. Maultzsch, C. Thomsen, Phys. Status Solidi B 246 (2009) 2577.

[9] S. Malola1, H. Häkkinen, P. Koskinen, Eur. Phys. J. D 52 (2009) 71.

[10] R. Gillen, M. Mohr, J. Maultzsch, Phys. Rev. B 81 (2010) 205426.

[11] H. Bu a, Y.F. Chen, M. Zou, H. Yi, K.D Bi, Z.H Ni, Phys. Lett. A 373 (2009) 3359.

[12] M. Neek-Amal, F.M. Peeters, Phys. Rev. B 82 (2010) 085432.

[13] D.D. Quinna, J. P. Wilber, C.B. Clemons, G.W. Young, A. Buldum, Int. J. 
Non-Linear Mech. 42 (2007) 681.

[14] O. Frank, G. Tsoukleri, J. Parthenios, K. Papagelis, I. Riaz, R. Jalil, K.S. Novoselov,

C. Galiotis, ACS-Nano 4 (2010) 3131.

[15] M. Neek-Amal, F.M. Peeters, Appl. Phys. Lett. 97 (2010) 153118.

[16] Q. Lu, R. Huang, Phys. Rev. B 81 (2010) 155410.

[17] D.V. Kosynkin, A.L. Higginbotham, A. Sinitskii, J.R. Lomeda, A. Dimiev, B.K. Price, J.M. Tour, Nature 458 (2009) 872.

[18] F. Cataldo, G. Compagnini, G. Patané, O. Ursini, G. Angelini, P.R. Ribic, G. Margaritondo, A. Cricenti, G. Palleschi, F. Valentini, Carbon 48 (2010) 2596.

[19] J.M Cai, P. Ruffieux, R. Jaafar, M. Bieri, T. Braun, S. Blankenburg, M. Muoth, A.P. Seitsonen, M. Saleh, X.L. Feng, K. Müllen, R. Fasel, Nature 466 (2010) 470.

[20] D. Sen, K.S. Novoselov, P.M. Reis, M.J. Buehler, Small 6 (2010) 1108.

[21] Y.C. Fan, J.L. Li, X. Liu, L.J. Wang, X.H. Chen, S.K. Sun, A. Kawasaki, W. Jiang, Carbon 49 (2011) 1439.

[22] R.F. Gibson, E.O. Ayorinde, Y.F. Wen, Compos. Sci. Technol. 67 (2007) 1-28.

[23] M. Kisa, Compos. Sci. Technol. 64 (2004) 1391.

[24] C.N.R. Rao, A.K. Sood, K.S. Subrahmanyam, A. Govindaraj, Angew. Chem. Int. Ed. 48 (2009) 7752. 
[25] F. Scarpa, S. Adhikari, R. Chowdhury, Phys. Lett. A 374 (2010) 2053.

[26] K. Behfar, R. Naghdabadi, Compos. Sci. Technol. 65 (2005) 1159.

[27] J.X. Shi, Q.Q. Ni, X.W. Lei, T. Natsuki, Comp. Mater. Sci. 50 (2011) 3085.

[28] J.X. Shi, Q.Q. Ni, X.W. Lei, T. Natsuki, J. Appl. Phys. 110 (2011) 084321.

[29] T. Natsuki, X.W. Lei, Q.Q. Ni, M. Endo, Phys. Lett. A 374 (2010) 2670-2674.

[30] M. Aydogdu, S. Filiz, Physica E 43 (2011) 1229.

[31] A.R. Setoodeh, M. Khosrownejad, P. Malekzadeh, Physica E 43 (2011) 1730.

[32] R. Ansari, H. Rouhi, S. Sahmani, Physica E 44 (2011) 373.

[33] S.C. Pradhan, Phys. Lett. A 373 (2009) 4182.

[34] A. Farajpour, M. Mohammadi, A.R.Shahidi, M.Mahzoon, Physica E 43 (2011) 1820.

[35] S.C. Pradhan, A. Kumar, Comp. Mater. Sci. 50 (2010) 239.

[36] S.C. Pradhan, J. K. Phadikar, Phys. Lett. A 373 (2009) 1062.

[37] R. Ansari, S. Sahmani, B. Arash, Phys. Lett. A 375 (2010) 53.

[38] R. Ansari, R. Rajabiehfard, B. Arash, Comp. Mater. Sci. 49 (2010) 831.

[39] S. Kitipornchai, X.Q. He, K.M. Liew, Phys. Rev. B 72 (2005) 075443.

[40] J.N. Reddy, Int. J. Eng. Sci. 45 (2007) 288. 


\section{Figure captions}

Fig. 1 Analytical model of a DLGNR. (a) Continuum model of a DLGNR, (b) Cross-section of a DLGNR embedded in an elastic matrix.

Fig. 2 In-phase and anti-phase vibration modes of DLGNRs with free supported boundary conditions.

Fig. 3 Relationship between the natural frequencies and vibration modes of DLGNRs of different aspect ratio with $e_{0} a=0 \mathrm{~nm}$. (a) In-phase modes, (b) Anti-phase modes.

Fig. 4 Nonlocal effects on the natural frequencies of DLGNRs with $L / b=10$ for different 
vibration modes. (a) In-phase modes, (b) Anti-phase modes.

Fig. 5 Nonlocal effects on the natural frequencies of DLGNRs of different aspect ratio for in-phase mode 1.

Fig. 6 Relationship between natural frequencies and vibration modes of DLGNRs embedded in different elastic matrices with $e_{0} a=0 \mathrm{~nm}$ and $L / b=10$.

Fig. 1

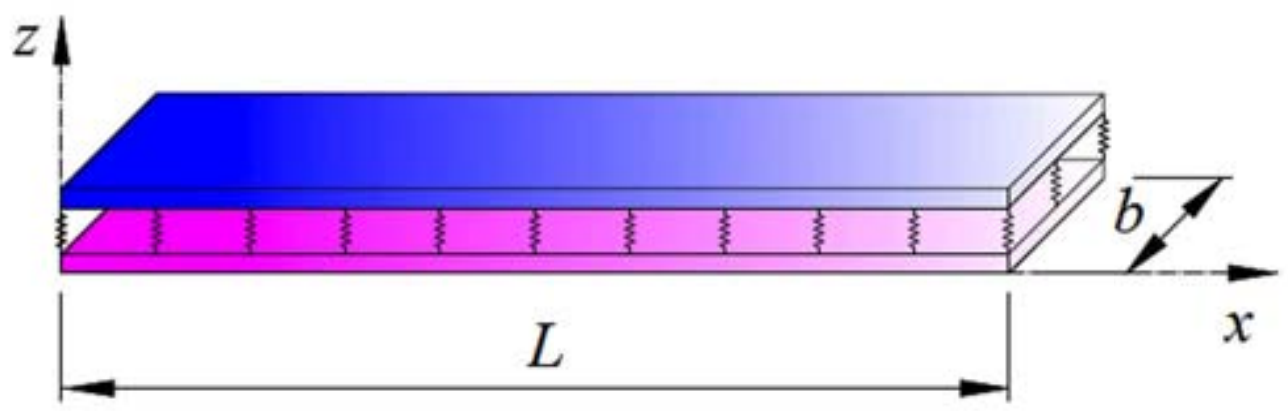

(a) 


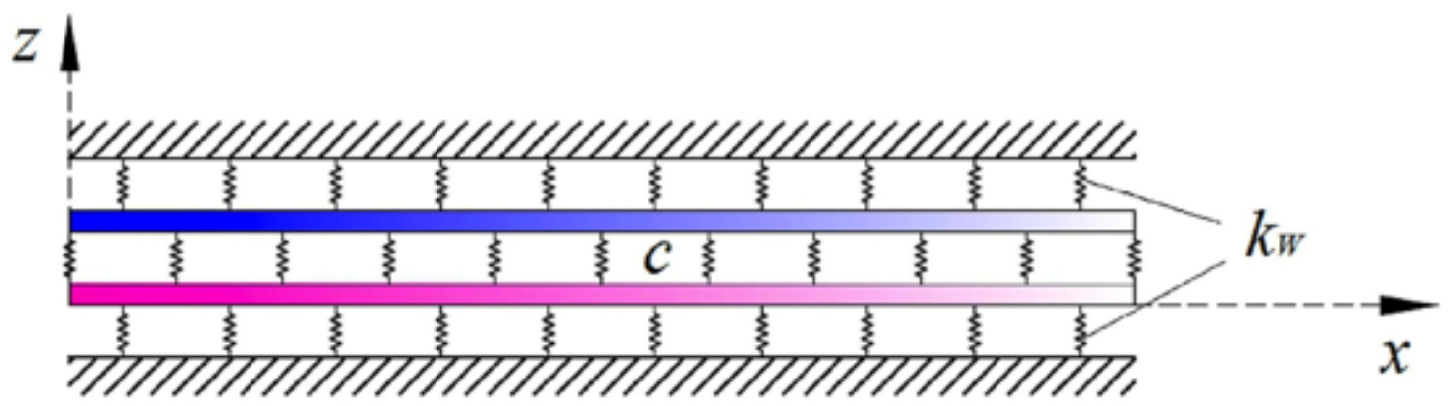

(b)

Fig. 2 


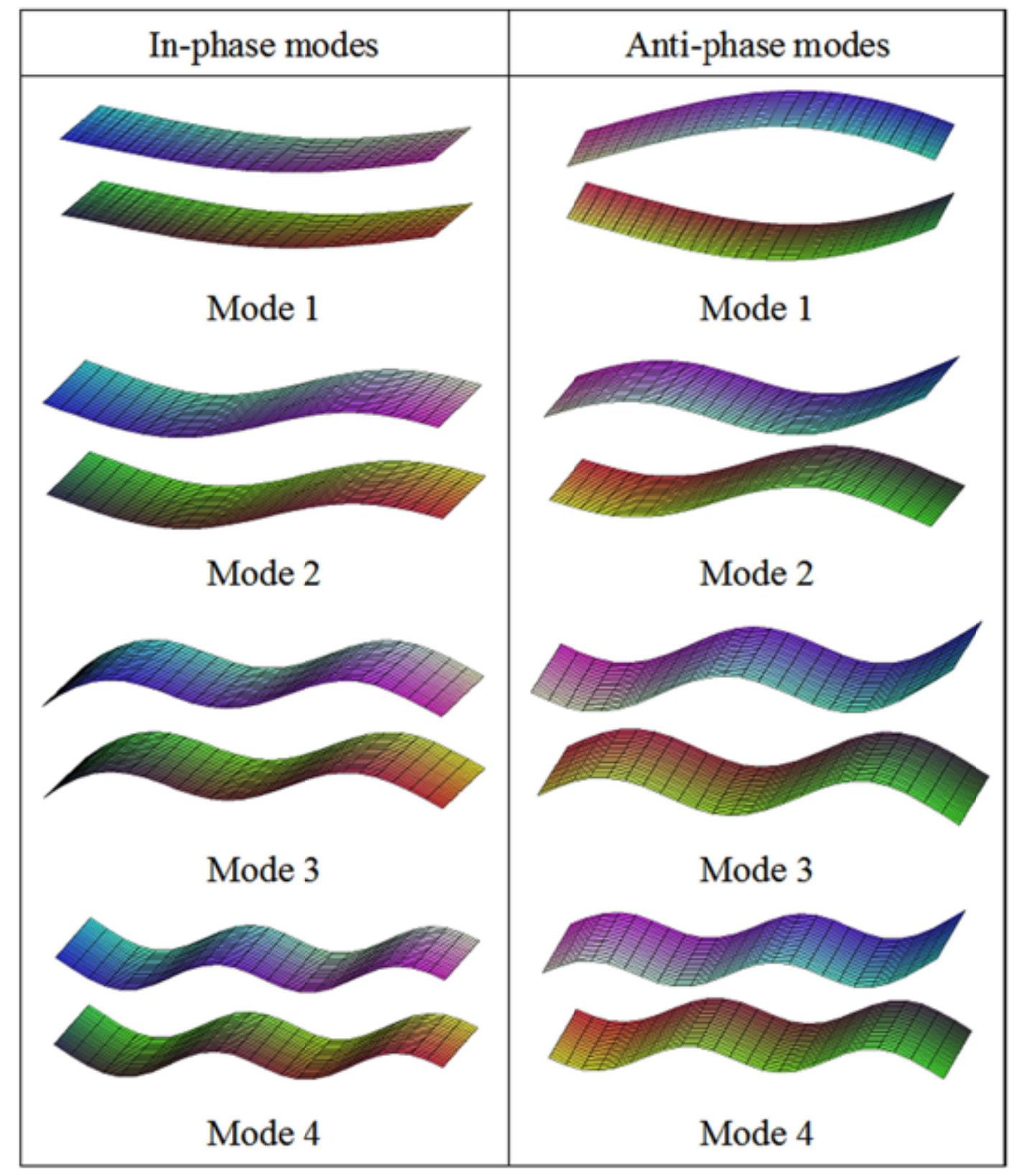

Fig. 3 

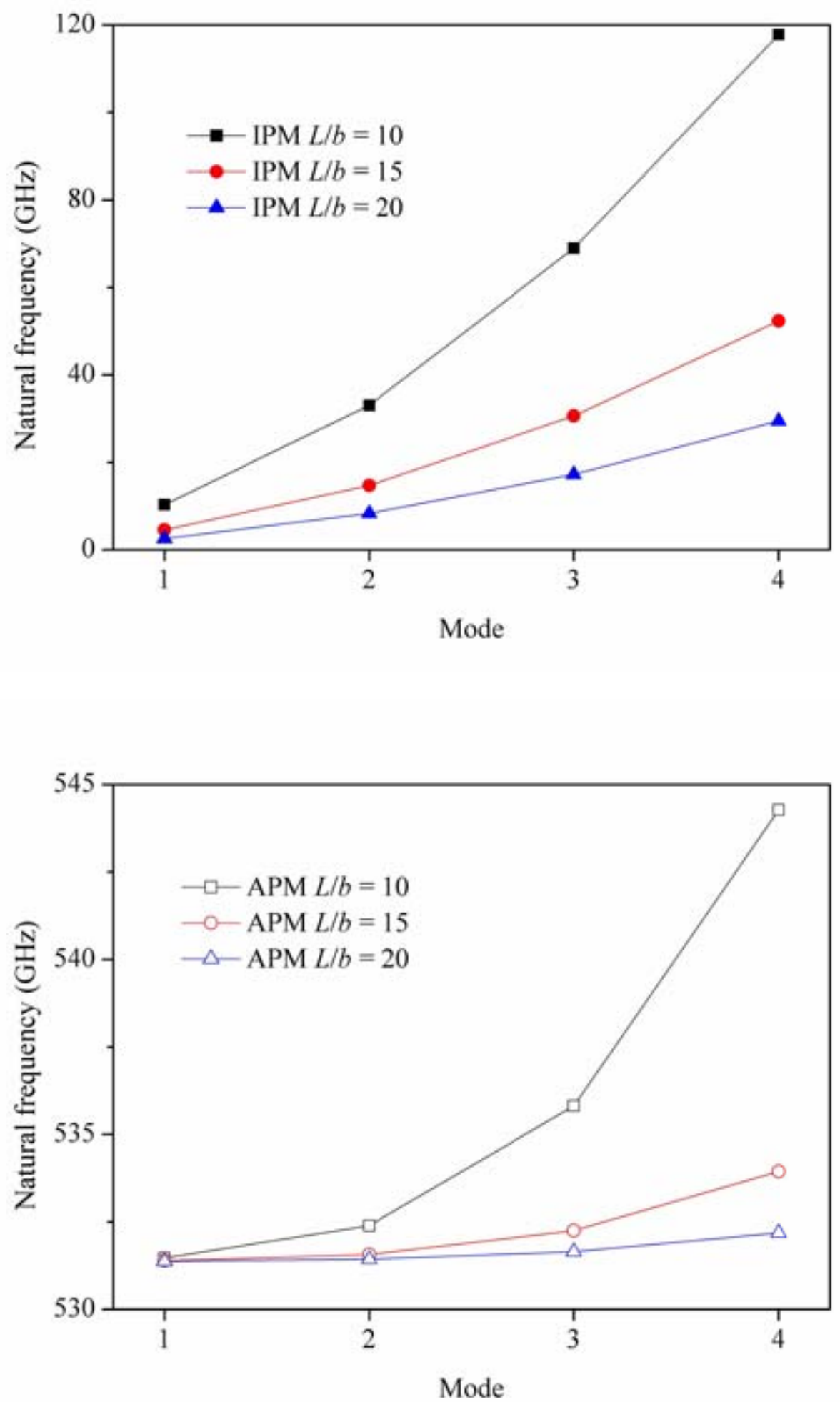

Fig. 4 

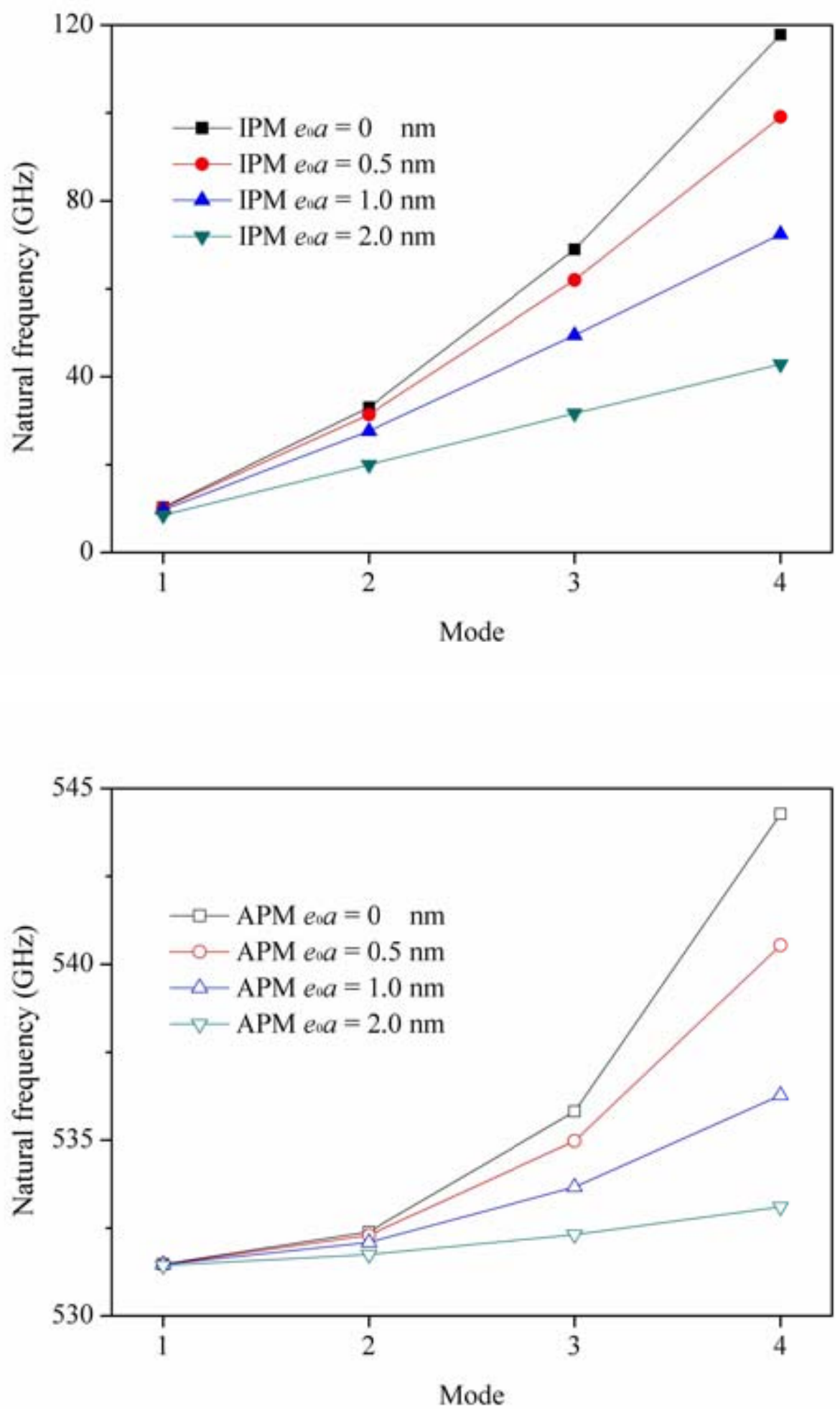

Fig. 5 


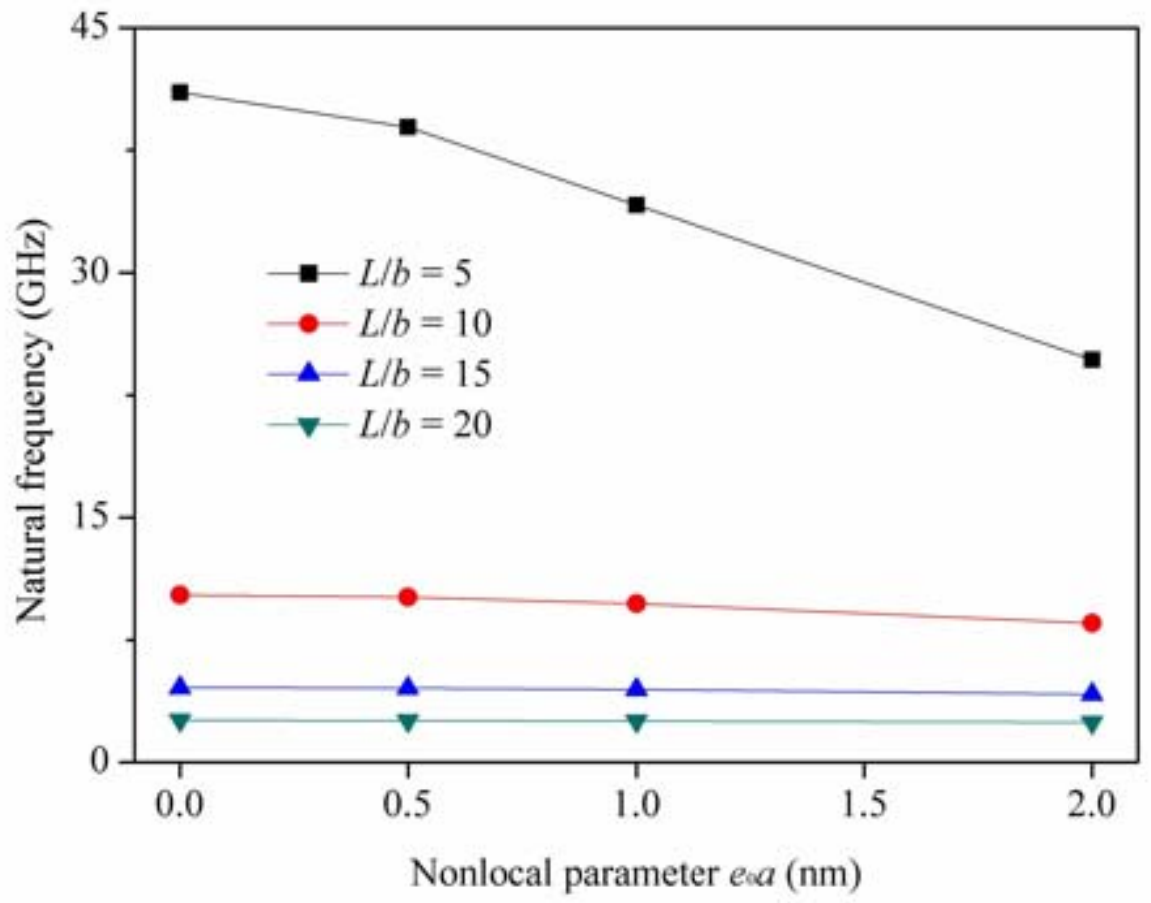

Fig. 6 


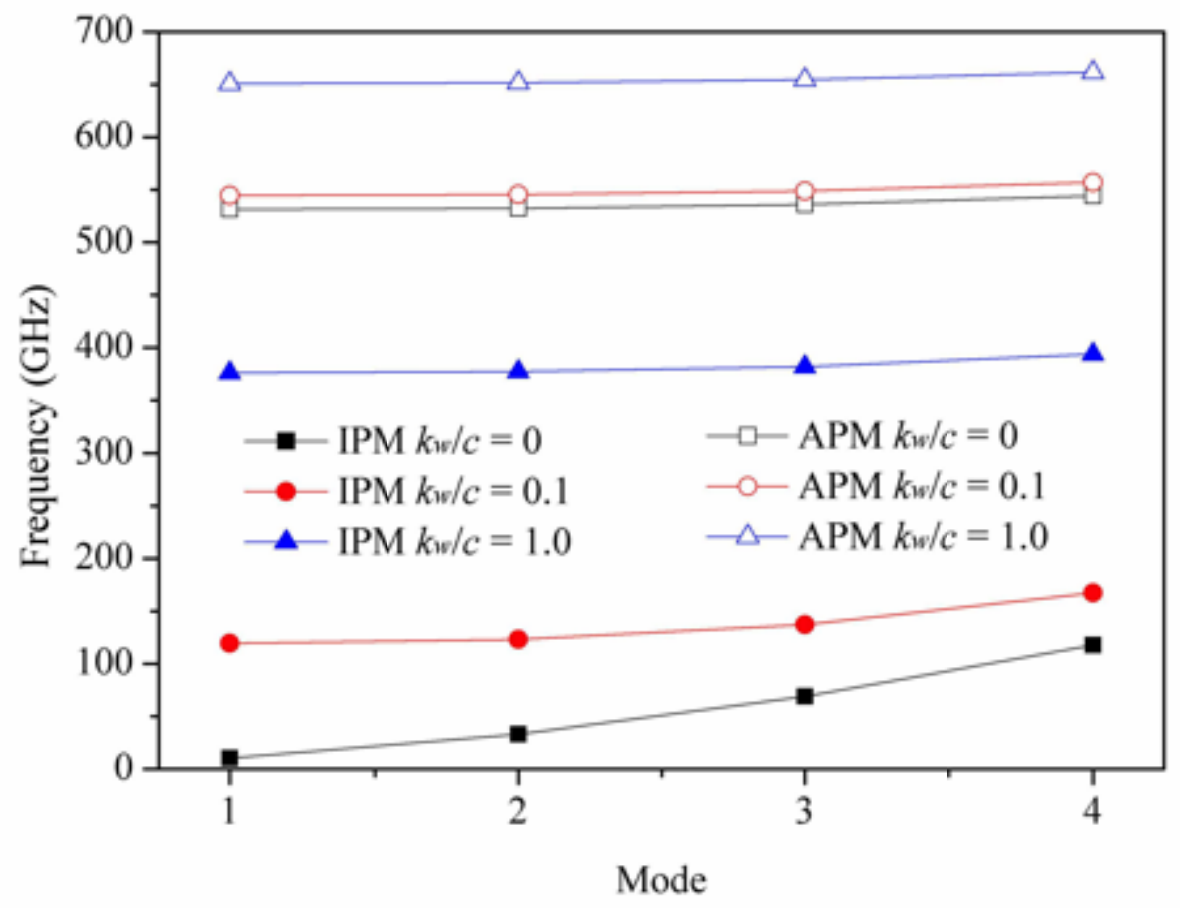

